\title{
Chaotic behaviour of an electrical analogue to the mechanical double pendulum
}

www.jestr.org

\author{
M. P. Hanias * \\ Chalkis Institute of Education and Technology, GR 34400, Evia, Chalkis. \\ Received 4 January 2008; Accepted 20 February 2008
}

\begin{abstract}
In this paper the analogy between a mechanical double pendulum and an oscillating electrical system is presented. Instead of using analytic equations, we used the MultiSim circuit simulation environment in order to reproduce and interpret the response of the electrical oscillator. The electrical double pendulum presents a chaotic regime which is studied quantitatively by means of state space reconstruction. For this purpose the optimal delay time is calculated and the minimum embedding dimension is found with the method of False Nearest Neighbors.
\end{abstract}

Keywords: mechanotronic, Chaos, Time Series

\section{Introduction}

Analogies between mechanical and electrical systems have attracted quite some interest in recent years [1-3]. The natural sine-wave oscillation for a mechanical system is called Simple Harmonic Motion. The same underlying principles govern both the oscillation of a capacitor/inductor circuit and the action of a pendulum, hence the similarity in effect. It is an interesting property of any pendulum that its periodic time is governed by the length of the string holding the mass, and not by the weight of the mass itself. That is why a pendulum will keep swinging at the same frequency as the oscillations decrease in amplitude. The oscillation rate is independent of the amount of energy stored in it. The same is true for the capacitor/inductor circuit. The rate of oscillation is strictly dependent on the sizes of the capacitor and inductor, not on the amount of voltage (or current) at each respective peak in the waves. It is well known that two coupled simple pendulums constitute a double pendulum which can exhibit chaotic behavior [4]. We propose that coupled L-C circuits can produce chaotic voltage oscillations as well. For this purpose, we have selected to use Multisim [5] as a circuit simulator, since it provides an interface as close as to the real implementation environment. In addition, complete circuits schematics and oscilloscope graphical plots are presented.

\section{Coupled LC-Circuits simulation}

Consider the pendulum of fig. 1 with mass $\mathrm{m}_{1}$ connected by a rigid mass-less wire of length $l_{1}$ to a fixed reference. The angle is denoted by $\theta_{1}$, and the gravity constant by $\mathrm{g}$.

*E-mail address: mhanias@teihal.gr

ISSN: 1791-2377 @ 2008 Kavala Institute of Technology. All rights reserved.
Now, we indent to study a full nonlinear analogue of the pendulum to an L-C electrical circuit, where we consider masses as inductive elements.

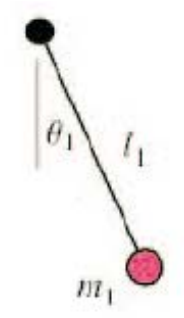

Fig. 1 Single mechanical pendulum.

Consider the electrical circuit that is shown in Fig. 2. We consider the rotational force resulting from gravity as a capacitive element. In the analogy, this means that this will result in a capacitor and a corresponding voltage.

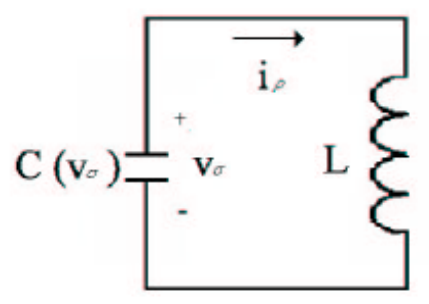

Fig. 2 Single pendulum equivalent LC circuit 


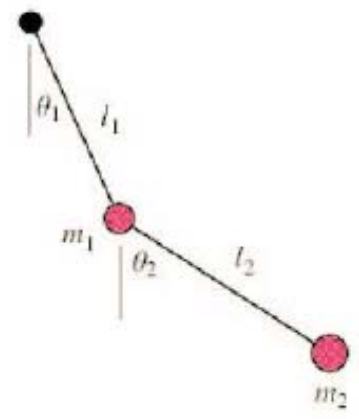

Fig. 3 Mechanical double pendulum

Now we can consider a double pendulum with masses $\mathrm{m}_{1}, \mathrm{~m}_{2}$ and rigid mass-less wires of lengths $l_{1}$ and $l_{2}$. The angles with the vertical are denoted by $\theta_{1}$ and $\theta_{2}$, as illustrated in Fig. 3 . The equivalent electric circuit is shown in Fig. 4,

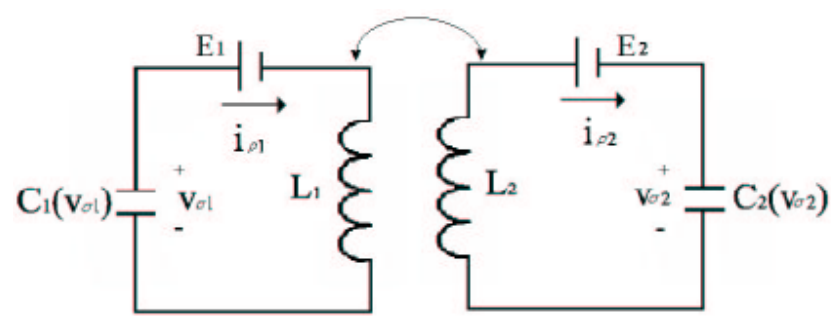

Fig. 4 Double pendulum equivalent $\mathrm{C}-\mathrm{L}$ electrical circuit

where inductance $\mathrm{L}$ is the electric analogue of mass $\mathrm{m}$ and capacitance $\mathrm{C}$ is electric analogue of 1 .

\section{Coupled LC-Circuits simulation}

We have selected to use Multisim to simulate the double pendulum equivalent $\mathrm{C}-\mathrm{L}$ electrical circuit, since it provides an interface as close as to the real implementation environment. The simulated circuit is shown at Fig. 5.

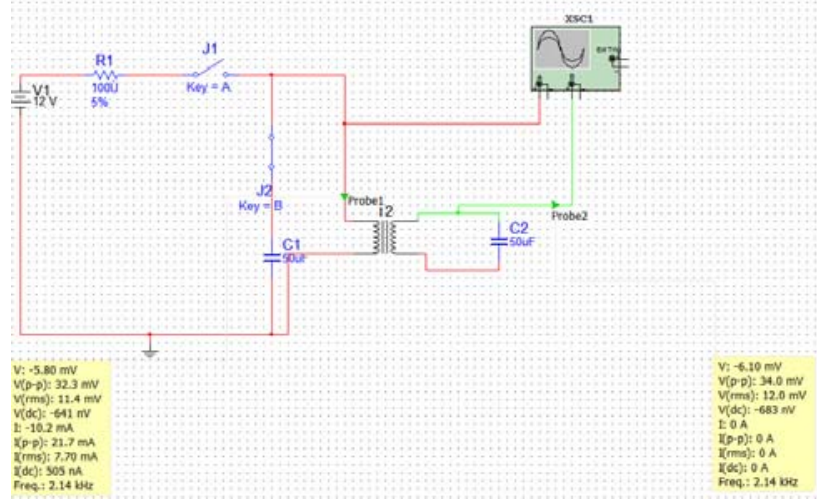

Fig.5 Multisim simumation of a mechanical double pendulum

When the switch $\mathrm{J} 1$ is closed the capacitor $\mathrm{C}_{1}$ is charged then the switch opens again and the circuit $\mathrm{L}_{1}, \mathrm{C}_{1}$ oscillates. We used an ideal transormer with primary coil inductance $\mathrm{L}_{1}$ and secondary coil inductance $L_{2}$ and a coefficient of coupling $\mu$, so the influence of each inductance is interpeted by the rms voltage drop acroose it, namely $\mathrm{U}_{1}$ and $\mathrm{U}_{2}$. When the values of the circuit elements are $\mathrm{C}_{1}=50 \mu \mathrm{F}, \mathrm{C}_{2}=50 \mu \mathrm{F}, \mathrm{L}_{1}=\mathrm{L}_{2}=10 \mu \mathrm{H}$, and $\mu=0.1$, then $U_{1}=11.4 \mathrm{mv}, \mathrm{U}_{2}=12 \mathrm{mv}$ and periodic oscillations occur, as shown at Fig. 6. This is the electrical analogue of a mechanical pendulum when $\mathrm{m}_{1}=\mathrm{m}_{2}$ and $\mathrm{l}_{1}=\mathrm{l}_{2}$

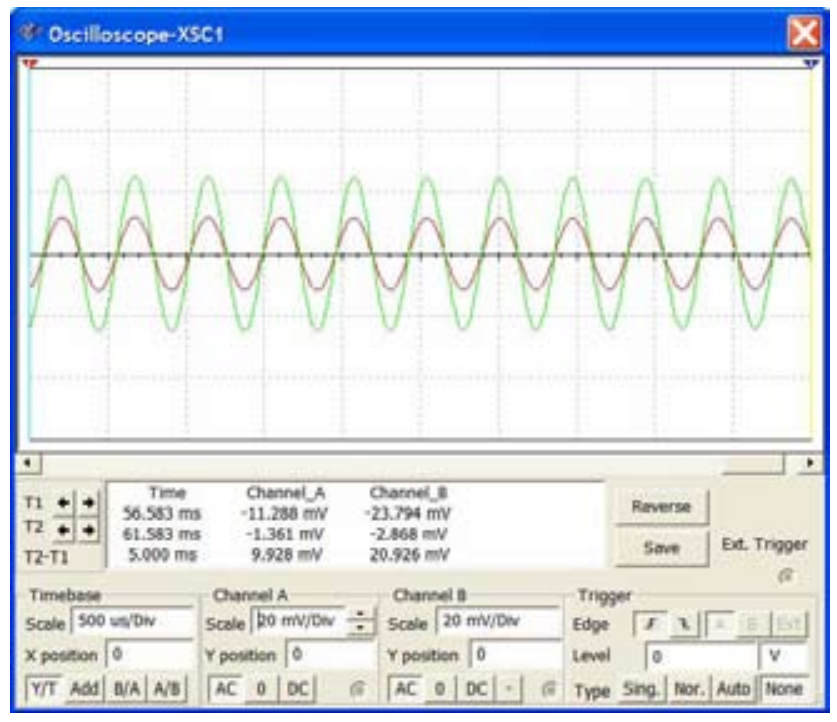

Fig.6 Periodic oscillations (Voltage at primary coil -red line,Voltage at secondary coil green line)

When we change the parameters to $\mathrm{C}_{1}=60 \mu \mathrm{F}, \mathrm{C}_{2}=60 \mu \mathrm{F}$ and $\mathrm{L}_{1}=10 \mu \mathrm{H}, \mathrm{U}_{1}=38.4 \mathrm{mv}, \mathrm{L}_{2}=10 \mu \mathrm{H}, \mathrm{U}_{2}=23 \mu \mathrm{V}$ and $\mu=0.001$, then the first circuit exhibits periodic oscillations (red line at Fig. 7), while voltage on the second circuit exhibits chaotic oscillations (Fig. 7 green line). This is the electrical analogue of a mechanical pendulum entering in the chaotic regime, when $\mathrm{m}_{1}>>\mathrm{m} 2$ and $\mathrm{l}_{1}=\mathrm{l}_{2}$.

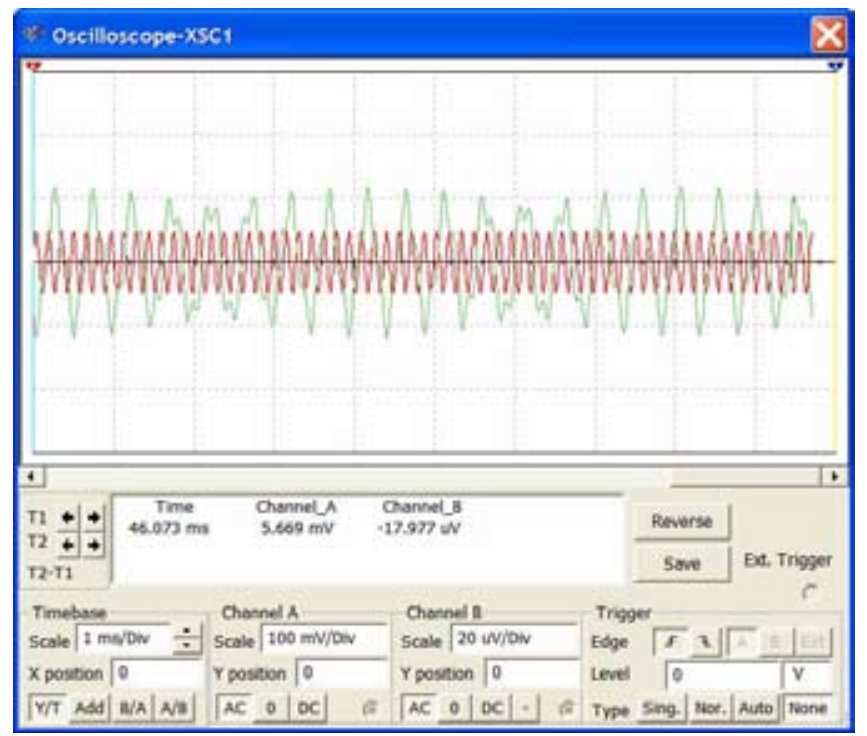

Fig. 7 Periodic oscillations of voltage at primary coil (red line) and chaotic oscillations of voltage at secondary coil (green line).

With $\mathrm{C}_{1}=60 \mu \mathrm{F} \quad \mathrm{C}_{2}=60 \mu \mathrm{F}$ and $\mathrm{L}_{1}=10 \mu \mathrm{H}, \mathrm{U}_{1}=31.3 \mathrm{mv}$, $\mathrm{L}_{2}=10 \mu \mathrm{H}, \mathrm{U}_{2}=10.4 \mathrm{mV}$ and $\mu=0.5$, then both circuits exhibit 
chaotic oscillations as shown at Fig. 8. This is the electrical analogue of a mechanical pendulum when $\mathrm{m}_{1}=3 \mathrm{~m}_{2}$ and $\mathrm{l}_{1}=\mathrm{l}_{2}$

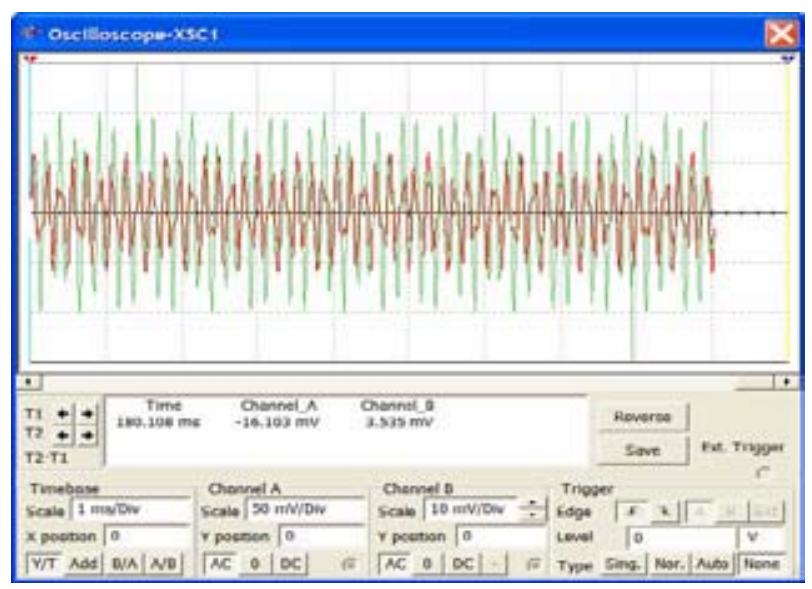

Fig. 8 Chaotic oscillations (Voltage at primary coil -red line,Voltage at secondary coil green line)

\section{State space reconstruction}

To analyze quantatively the chaotic data of circuit at Fig. 8 we record $\mathrm{N}=10000$ points of the voltage oscillations at primary and secondary coil. At Fig. 9 part of voltage oscillations at primary coil are shown while at Fig. 10 are part of voltage oscillations at secondary coil are shown.

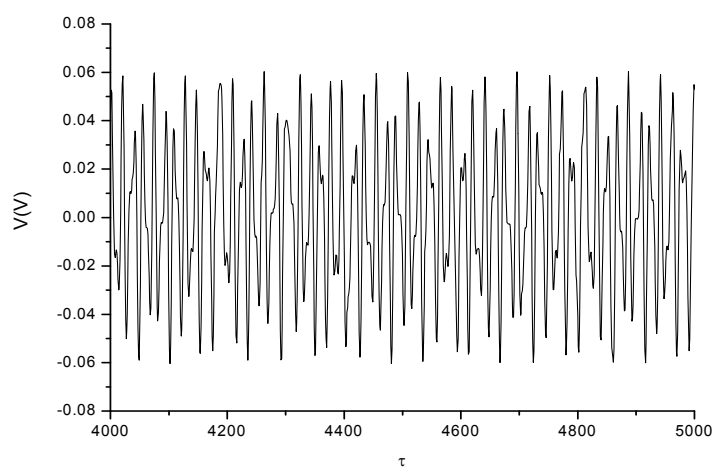

Fig. 9 Chaotic oscillations at primary coil

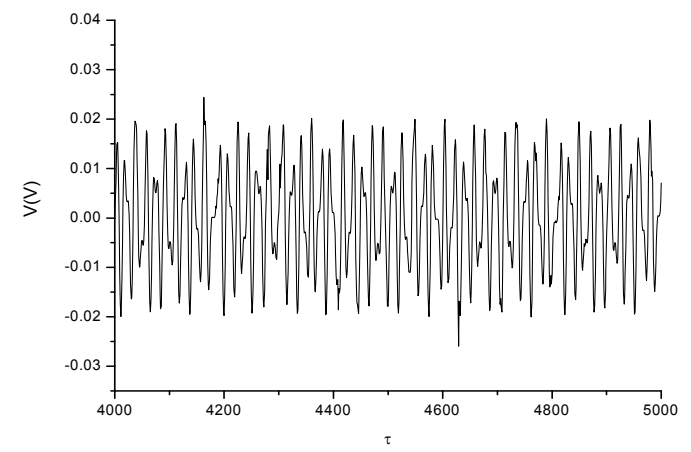

Fig. 10 Chaotic oscillations at secondary coil
From our data we construct a vector $\boldsymbol{y}(t(i)), i=1$ to $\mathrm{N}$ in the $m$ dimensional phase space given by the following relation $[6,7]$

$$
\mathbf{y}(t(i))=(x(t(i)), x(t(i+\tau)), \ldots, x(t(i+(m-2) \tau)), x(t(i+(m-1) \tau)))
$$

This vector, represents a point to the $\mathrm{m}$ dimensional phase space in which the attractor is embedded each time, where $\tau$ is the time-delay $\tau=\mathrm{i} \Delta \mathrm{t}$ and $\Delta \mathrm{t}$ is the sample rate $\Delta \mathrm{t}=6.25 \times 10^{-6} \mathrm{~s}$. $x(t(i))$ represents a value of the examined scalar time series in time, in our case the voltage at L1 and L2 respectively, corresponding to the $i$-th component of the time series. Use of this method reduces phase space reconstruction to the problem of proper determining suitable values of $m$ and $\tau$. The choice of these values is not always simple, especially when we do not have any additional information about the original system and the only source of data is a simple sequence of scalar values, acquired from the original system. The dimension, where a time-delay reconstruction of the phase space provides the necessary number of coordinates is called embedding dimension $m$.

\subsection{Time delay $\tau$}

Using the average mutual information we can obtain $\tau$, less associated with linear point of view, and thus more suitable for dealing with nonlinear problems. The average mutual information, which may be expressed by the following formula $[8,9]$ :

$$
I(\tau)=\sum_{x(t(i)), x(t(i+\tau))} P(x(t(i)), x(t(i+\tau))) \log _{2}\left(\frac{P(x(t(i)), x(t(i+\tau)))}{P(x(t(i))) P(x(t(i+\tau)))}\right)
$$

$P(x(t(i)), x(t(i+\tau)))$ is the joint probability density for the values $x(t(i))$ and $x(t(i+\tau))$, while $P(x(\tau))$ expresses the probability density of the value $x(\tau(i))$. In general, $I(\tau)$ expresses the amount of information (in bits), which may be extracted from the value in time $t(i)$ about the value in time $t(i+\tau)$. A value of $\tau$, suitable for the phase space reconstruction, is usually considered the position of the first minimum of $I(\tau)$ [6]. The timedelay is determined by the first minimum of the mutual information [6]. In this case, $\tau=4$ time steps for both time series measured at primary and secondary coils, as shown in Fig. 11 and 12 respectively.

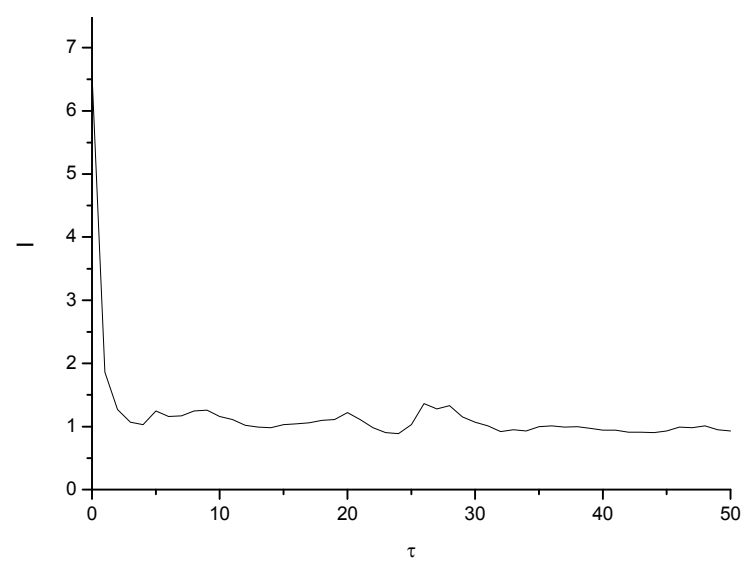

Fig. 11: Mutual Information I vs. time delay $\tau$ for time series at primary coil of Fig. 9 


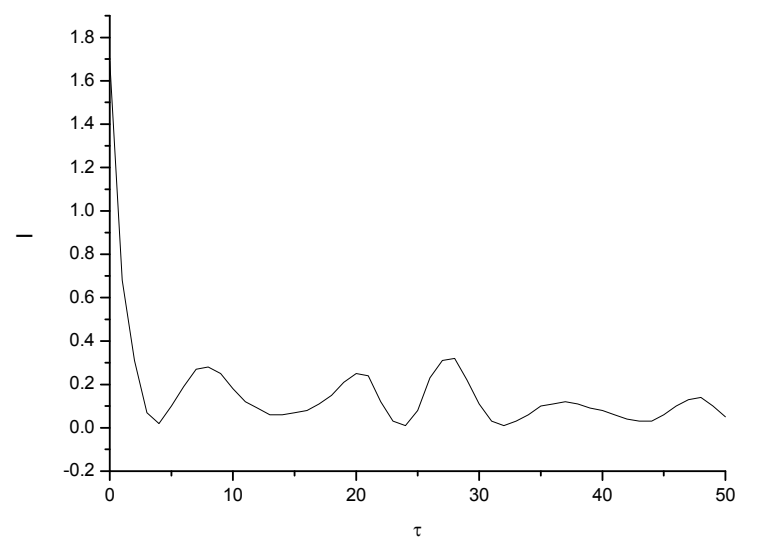

Fig. 12: Mutual Information I vs. time delay $\tau$ for time series at secondary coil of fig. 10

\subsection{Embedding dimension $\mathrm{m}$}

After obtaining the satisfactory value of $\tau$, the embedding dimension $m$ is to be determined in order to finish the phase space reconstruction. For this purpose we use the method of False Nearest Neighbors [6, 10]. More specifically, the method is based on a fact that when embedding dimension is too low, the trajectory in the phase space will cross itself. If we are able to detect these crossings, we may decide whether the used $m$ is large enough for correct reconstruction of the original phase space (i.e. when no intersections occur) or not. When intersections are present for a given $m$, the embedding dimension is too low and we have to increase it at least by one. Then, we test the eventual presence of self-crossings again $[8,10]$. The practical realization of the described method is based on testing of the neighboring points in $m$-dimensional phase space. Typically, we take a certain amount of points in the phase space and find the nearest neighbor to each of them. Then we compute distances for all these pairs and also their distances in $(m+1)$-dimensional phase space. The rate of these distances is given by

$$
P=\frac{\left\|\mathbf{y}_{i}(m+1)-\mathbf{y}_{n(i)}(m+1)\right\|}{\left\|\mathbf{y}_{i}(m)-\mathbf{y}_{n(i)}(m)\right\|}
$$

Where $\mathbf{y}_{\mathrm{i}}(m)$ represents the reconstructed vector, belonging to the $i$-th point in the $m$-dimensional phase space and index $n(i)$ denotes the nearest neighbour to the $i$-th point. If $P$ is greater than some value $P_{\max }$, we call this pair of points false nearest neighbors (i.e. neighbors, which arise from trajectory self-intersection and not from the closeness in the original phase space ). In the ideal case, when the number of false neighbors falls to zero, then the value of $m$ is found. For this purpose we compute the rate of false nearest neighbours in the reconstructed phase space using the formula

$$
\left|x_{i+m \tau}-x_{n(i)+m \tau}\right| \geq R_{A}
$$

where $R_{A}$ is the radius of the attractor,

$$
R_{A}=\frac{1}{N} \sum_{i=1}^{N}\left|x_{i}-\bar{x}\right|
$$

and

$$
\bar{x}=\frac{1}{N} \sum_{i=1}^{N} x_{i}
$$

is the average value of time series.

When the following criterion

$$
P \geq P_{\max }
$$

is satisfied then it can be used to distinguish between true and false neighbours [8]. The dimension $m$ is found when the percent of false nearest neighbors decreases below some limit, typically set to $1 \%$ [11] so we choose $\mathrm{P}_{\max }=10$. We used Matlab code to calculate the mutual information I and the quantity $\mathrm{P}$ The percentage of false neighbors that is under the above limit is achieved for $m=4$, for both time series thus this value should be suitable for the purpose of phase space reconstruction. This is shown at Figs. 13, 14 for our time series at the primary and secondary coil respectively.

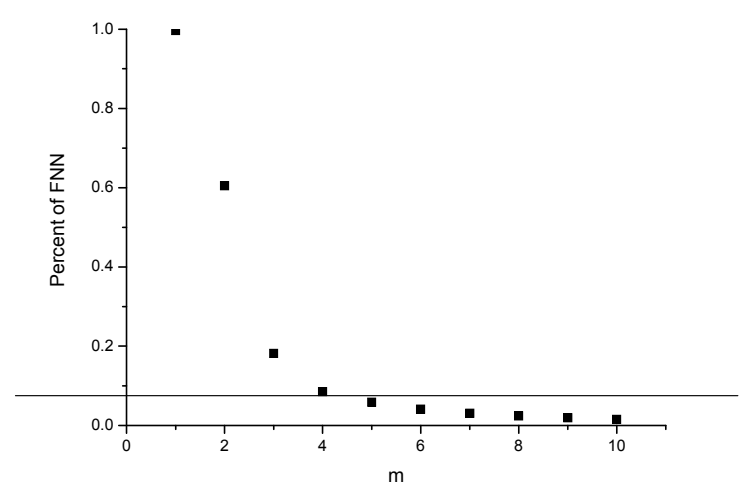

Fig. 13: Percent of false nearest neighbors number FNN vs. $m$ for time series at primary coil.

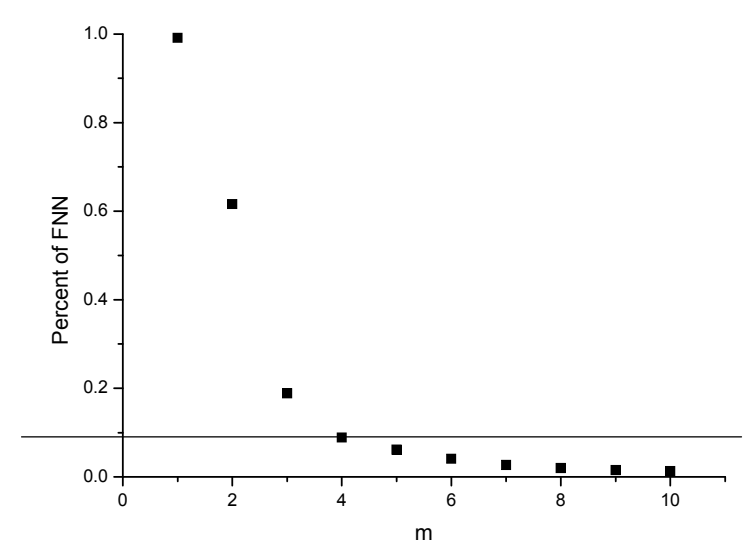

Fig. 14: Percent of false nearest neighbors number FNN vs. $m$ at secondary coil. 


\section{Conclusion}

In this paper we have presented the electrical interpretation of a mechanical chaotic double pendulum. The L-C coupled circuits exhibit the analogue chaotic behavior when we choose values of $\mathrm{L}, \mathrm{C}$ that resembles the length and mass of mechanical pendulum. We found the minimum embedding dimension with the method of False Nearest Neighbors to be $m=4$. This means that we need four degrees of freedom or four differential equations to describe the state space of the system as is also the case in the problem of the mechanical double pendulum.

\section{References}

1. M. Smith, "Synthesis of mechanical networks: the interter," IEEE Trans. Aut. Contr., vol. 47, no. 10, pp. 1648-1662, October (2002).

2. S. Stramigioli, "Modeling and IPC Control of Interactive Mechanica

3. Systems: a coordinate free approach.”, Springer Verlag, London, (2000).

4. Alessandro de Rinaldis and Jacquelien M.A. Scherpen, "An Electrical Interpretation of Mechanical Systems via the Pseudo-inductor in the Brayton-Moser Equations"Proceedings of the 44th IEEE Conference on Decision and Control, and the European Control Conference 2005 Seville, Spain, December 12-15, (2005)

5. R.B. Levien, S.M. Tan, Am. J. Physics, Vol 61, No 11, (1993)

6. K. E. Lonngren, "Notes to accompany a student laboratory experiment on chaos," IEEE Transactions on Education, Vol. 34, No.1, February (1991).
7. Kantz H. and T.Schreiber "Nonlinear Time Series Analysis", Cambridge University Press, Cambridge (1997)

8. Takens F. "Detecting strange attractors in turbulence", Lecture notes in Math., vol. 898, Springer, New York (1981)

9. Abarbanel H.D.I. "Analysis of observed chaotic data", Springer, New York, p.272 (1996)

10. Fraser A.M., Swinney H.L." Independent coordinates for strange attractors from mutual information", Phys. Rev. A, 33, 1134,(1986)

11. Kennel M.B., Brown R., Abarbanel H.D.I. "Determining embedding dimension for phase-space reconstruction using a geometrical construction", Phys. Rev. A, 45, 3403, (1992)

12. Kugiumtzis D., Lillekjendlie B., Christophersen N. (1994): Chaotic time series, Part I, Modeling Identification and Control 15, 205 